\title{
Contribution à l'étude des crues
}

\section{A contribution to the study of floods}

\author{
PAR I. LARRTEU
}

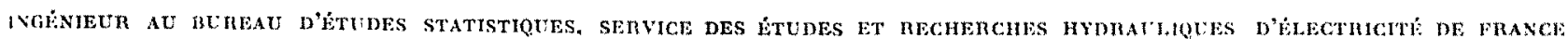

\begin{abstract}
Parmi les differentes méthodes d'estimation des crues a partir des précipitalions, on atilise surtout en France celles qui font appel aux résultats de crues réelles dija observées. Ces méthodes s'appuient sur la mème notion: L'opératenr permettant de passer des pluies aux débits est linéaire dans le temps et dans l'espace. Dans la méthode exposée cídessons nous avons repris cet axiome mais l'avons exploité d'une autre façon que nos devanciers.

Les résultats obtenus sur la rivière la Durance sont très encourageants et ils jettent une lumière nouvelle sur le mécanisme des crues pluviales. L'avenir nous apprendra peut-être si la construction des hydrogrammes synthétiques outre son róle de prévision, peut faire avancer l'analyse des crues.
\end{abstract}

La détermination des crues à partir des précipitations a fait lobjel de nombreuses études aussi bien en France qu'à l'étranger. La matière est tellement riche et tellement complexe qu'elle se prête à des analyses très variées.

Un certain nombre de conférenciers ont déjà brillamment exposé ici même, devant le Comité Technique de la Société Hydrotechnique de France, les idées générales et des applications fort intéressantes sur la question.

Nous désirons apporter notre contribution à ces recherches en indiquant les résultats que nous avons obtenus au cours d'une étude entamée depuis un certain temps.

\section{$*$}

Lorsqu'il pleut sur un bassin de réception, le débit augmente à l'exutoire du bassin, il $y$ a crue; c'est là un fait d'expérience trivial. Le relevé continu des débits au cours de la crue constitue l'hydrogramme de cette dernière. II se présente généralement sous la forme repré-

\begin{abstract}
Among the different methods of estimating foods caused by rainfall, those used in France usually meke use of the recordings of floods already observed. These methods depend on the same assumption: the operator allowing the transition from rainfall to discharge is a linear function of time and space. In the method explained below we have adopted this axiom but we have exploited it differently, as we explain.

The results obtained on the Durance Riber are very encouraging and they throw new light on the mechanism of floods caused by rainfall. The future will perhaps teach us whether the construction of synthetic hydrograms beyond its role of forecasting can bring about adbances in the analysis of floods.
\end{abstract}

sentée figure 1, quand il y a cu averse continue. On conçoit aisément que si la pluie ne s'était pas produite, le débit aurait suivi la ligne pointillée $\mathrm{A} \mathrm{B}^{\prime} \mathrm{C}^{\prime}$ (courbe de tarissement). D'autre part, la ligne BC est la continuation d'une nou-

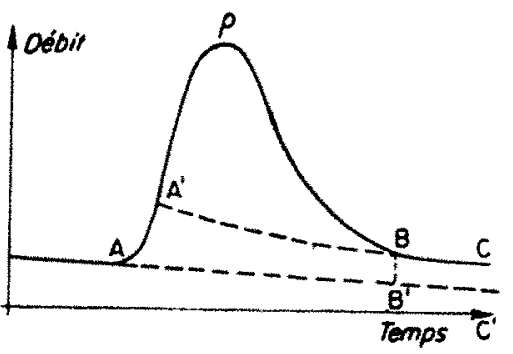

Fia. 1.

velle courbe de tarissement $\mathrm{A}^{\prime} \mathrm{BC}$. On est donc amené à considérer que la portion de l'hydrogramme due réellement au ruissellement de la pluie est comprise entre deux limites extrêmes représentées par les contours APBB' et $A^{\prime} P B$. Le volume $\mathrm{AA}^{\prime} \mathrm{BB}^{\prime}$ est relatif à des apports com- 
plexes, écoulement hypodermique, influence de la nappe phréatique.

Si nous voulons calculer un hydrogramme de ruissellement à partir des pluies en l'ajustant sur l'hydrogramme réel observé, il se pose un premier problème: détermination de Ihydrogramme pluie ruisselée. Ce n'est pas là chose facile, puisqu'on ne possède guère d'élément caractéristique pour l'isoler objectivement de l'hydrogramme total observé. Un problème analogue se pose pour les précipitations. Ces dernières ne contribuent pas en totalité au ruissellement.

Elles se divisent en plusieurs parties.

1. Un certain pourcentage s'évapore avant d'avoir le temps de s'écouler.

2. Une autre partie alimente la nappe phréatique.

3. Une autre va donner naissance à l'écoulement hypodermique.

4. Enfin une certaine portion, celle qui nous intéresse, ruisselle et contribue à la crue proprement dite.

Le problème se complique encore du fait que ces dillérentes parties ne sont pas en proportions fixes ni au cours de la crue, ni au cours des différentes saisons.

Considérons par exemple l'évaporation. Elle est beaucoup plus forte en été qu'en hiver, au début de la crue qu'à la fin. De même l'apport à la nappe phréatique, considérable au début de la crue, s'amenuise progressivement.

Il est extrêmement difficile d'évaluer les importances respectives des éléments 1,2 et 3 . Le quotient entre le volume de la crue ruisselée (si tant est qu'on puisse le déterminer exactement) et le volume précipité donne en efret un coefficient qui intègre globablement 1,2 et 3 .

En particulier, jusqu'à présent on n'avait pas mesuré effectivement l'importance de l'évaporation.

\section{CHOIX D'UNE MÉTHOdE}

L'utilisation des pluies pour la détermination des crucs n'est pas chose récente. Nombre d'hydrologues ont ainsi cherché à relier cause à effet.

Les méthodes utilisées qui supposent toutes la connaissance des précipitations, se divisent en deux groupes principaux.

Nous laisserons de côté celles du premier groupe qui font appel aux propriétés géographiques du bassin.

Les autres methodes utilisent toutes plus ou moins implicitement des notions que nous allons rappeler maintenant. On considère un bassin de réception $S$ et son exutoire $J$. Une averse uni- forme sur $S$, de durée unité et d'intensité unite, donne naissance à une crue de forme invariable, bien déterminée. Le relevé continu des débits observés en $J$ durant la crue constitue l'hydrogramme unitaire, élément fondamental.

L'exploitation de cet hydrogramme unitaire repose sur deux autres axiomes:

a) Une pluie d'intensité I mais de durée unitaire donne naissance à un hydrogramme déduit de l'hydrogramme unitaire en multipliant par I toutes les ordonnées;

b) Une pluie de durée non unitaire donne naissance à un hydrogramme résultant de l'addition d'hydrogrammes unitaires avec décalage dans le temps.

Ainsi la connaissance de l'hydrogramme unitaire et cel.e des pluies suffisent à déterminer l'hydrogramme de n̈importe quelle crue.

Ce schéma est séduisant surtout par sa simplicité théorique, malheureusement il se heurte à des objections, particulièrement en ce qui concerne le premier axiome, point faible de la méthode.

Ce premier axiome donne lieu à deux critiques, la première de nature théorique, la seconde de nature pratique.

La première concerne l'hypothèse d'un hydrogramme unitaire de forme invariable. Cette hypothèse a souvent été attaquée, mais il faut reconnaitre qu'en général, elle est assez bien vérifiée, du moins pour les petits bassins.

La seconde critique concerne la difficulté de rencontrer les conditions imposées : averse uniforme sur tout le bassin, d'intensité unité, de durée unité.

C'est pourquoi les hydrologues ont fait porter leurs principaux efforts sur la détermination des hydogrammes unitaires.

On peut suivre deux voies bien différentes pour aboutir à ce résultat :

Ou bien on attend patiemment une averse d'intensité constante, de durée suffisamment brève pour donner naissance à un hydrogramme unitaire. On risque alors d'attendre longtemps, car il est fort rare d'obtenir une averse uniforme dans le temps et dans l'espace sur un bassin de quelque grandeur. Aussi, en général, cette méthode de l'hydrogramme unitaire expérimental n'est utilisée que pour les tout petits bassins de nature très homogène.

Ou bien on cherche à déterminer l'hydrogramme unitaire à partir d'hydrogrammes quelconques $\left(^{*}\right)$. C'est le procédé utilisé pour les grands bassins. En particulier, une méthode con-

(*) Voir a ce sujet : Applied Hydrology, par JohNstone et Cross. 
siste à supputer un hydrogramme unitaire $a$ priori; à partir de ce dernier on construit l'hydrogramme d'une crue effectivement observée el l'on compare le résultat calculé à l'hydrogramme réel.

On corrige ensuite l'hydrogramme unitaire considéré au moyen des écarts observés, puis on recommence. Il y a convergence de l'hydrogramme supputé vers l'hydrogramme unitaire.

Cette méthode est souvent laborieuse et la convergence ne s'effectue pas toujours d'une façon très satisfaisante si l'hydrogramme de départ n'a pas été bien choisi.

Ces différentes considérations nous ont fait renoncer à l'utilisation des hydrogrammes unitaires proprement dit.

Nous utilisons un procédé que nous appelons méthode de l'hydrogramme élémentaire faute d'une désignation plus commode.

\section{MÉTHOdE DE L'HYDROGRAMME ÉLÉMENTAIRE}

Nous avons remplacé notre désignation antérieure d'hydrogramme synthétique par celle d'hydrogamme élémentaire parce que nous faisons appel dans notre analyse à des éléments de l'hydrogramme unitaire classique. C'est dire que notre méthode se rattache au premier des groupes indiqués plus haut.

Comme précédemment nous appliquons le principe fondamental suivant :

L'opérateur pluie-débit est linéaire à la fois dans le temps et dans l'espace.

Cela signifie concrètement qu'une suite d'averses successives produit une crue qui est la somme avec un décalage de temps convenable, des crues qu'aurait produites chaque averse séparément.

D'autre part, des averses produites sur diverses parties du bassin provoquent une crue qui est la somme des crues qu'aurait produit séparément chaque averse agissant seule.

Il ne faut pas se faire trop d'illusions sur la valeur théorique de ce principe. Contentons-nous du fait qu'en pratique, il se vérifie assez bien.

\section{ETABLISSEMENT DE LA FORMULE FONDAMENTALE}

Nous étudions un bassin de réception $S$, possédant une station de jaugeage à l'exutoire, et nous cherchons le debit $I(t)$ produit par une pluie donnce.

Pour établir l'équation fondamentale, nous allons maintenant nous placer à l'exutoire, à l'instant $t$; nous allons chercher la contribution d'un élément de surface $d S$ du bassin au débit entre les instants $t$ et $t+d t$. Le volume tombé dans la période $t-\tau-\theta, t-\tau-\theta+d \theta$ va donner la contribution suivante:

$$
h(\mathbf{P}, t-\tau-\theta) h(\mathbf{P}, t-\tau-\theta) d t d \mathrm{~S} r(\mathrm{P}, \theta) d 0
$$

Dans cette expression, : désigne le temps de parcours moyen de l'eau ruisselée entre le point $P$ situé dans $d S$ et l'exutoire, $h(P, t)$ la lame d'eau précipitée sur $d S$ entre $t$ et $t+d t, k(\mathbb{P}, t)$ le coefficient de ruissellement et $r(P, t)$ le coefficient d'étalement et de répartition de la pluie.

Pour obtenir la contribution totale de la zone $d s$, il suffit d'intégrer cetle expression par rapport à $\theta$ de 0 à $+\infty$. On oblient alors l'expression suivante :

$\int_{0}^{\infty} k(\mathbf{P}, t-\tau-\theta) h(\mathbf{P}, t-\tau-\theta) r(\mathbf{P}, 0) d t d \mathrm{~S} d \theta$

On aura le débit total à l'exutoire en intégrant encore une fois cette expression par rapport a $S$. Finalement, nous obtenons pour expression du débit à l'exutoire :

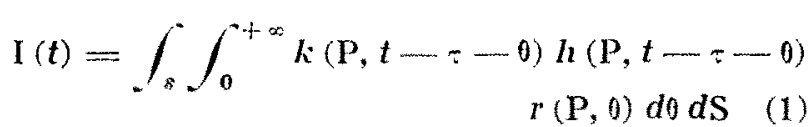

Il convient de faire ici le rapprochement entre l'hydrogramme unitaire tel que nous l'avons défini plus haut et l'hydrogramme que nous construisons à partir de l'expression (1). Il est facile de vérifier que l'expression :

$$
k(\mathrm{P}, t-\tau-0) r(\mathrm{P}, \theta)
$$

est l'élément différentiel spatial de l'hydrogramme unitaire. Par conséquent, sans utiliser directement ce dernier, nous le voyons apparaitre dans notre analyse.

Nous allons maintenant passer en revue les différents facteurs qui apparaissent dans la relation (1).

$$
\text { Facteun } h(\mathrm{P}, t-\tau-0) d t
$$

Il n'y a pas grand-chose à dire sur cetle quantité. Nous remarquerons seulement que, dans les applications, lintégration sera faite numériquement, ce qui nous obligera à posséder des relevés très rapprochés pour les pluies du bassin. Si par exemple on adople un $d t$ de six heures, il nous faudra obtenir des relevés toules les six beures,

L'idéal, d'ailleurs, consisterait à posséder un enregistrement continu des pluies, ce qui permettrait de choisir au mieux la longueur de l'élément de temps $d t$. Nous verrons plus bas que cet idéal a été obtenu dans les applications pratiques que nous avons faites.

Nous avons maintenant à considérer le temps de ruissellement $\tau=(d / v)$, où $v$ désigne la vitesse moyenne de ruissellement et $d$ la distance de parcours moyenne des gouttelettes d'eau. 
Nous supposons la vitesse moyenne $v$ constante. En réalité, il n'en est cerlainement rien; $v$ dépend à la fois du lieu (pente, consistance des terrains, etc.) et des circonstanees météorologiques (état de saturation du sol, de l'air, etc.). Il est à peu près impossible de tenir compte des différents facteurs qui entrent ainsi en jeu et on doit se contenter de considérer une vitesse moyenne constante au cours du temps.

Nous avons obtenu une valeur moyenne de $v$ a partir de la vitesse moyenne $V$ d'écoulement dans la rivière.

$$
\text { Factede } k(\mathbf{P}, t-=-0)
$$

Ce facteur a une interprétation physique très simple. Il représente le coefficient de ruissellement. Nous avons donc a priori quelques idées sur sa lorme. Il est évidemment compris entre 0 et 1 , et croissant avec le temps à partir du début de la pluie. Ceci posé, il est difficile d'aller plus loin pour lui chercher une expression mathématique guidée par des considérations physiques.

Nous utiliserons des formes mathématiques aussi simples que possible et n'introduirons qu'un seul paramètre. Nous pourrons éventuellement songer à faire varier ce paramètre suivant les saisons puisqu'il semble évident à première vue que $k(P, t-\tau-\theta)$ est plus grand en hiver qu'en été. Mais, seule l'expérience directe peut nous guider à ce stade de l'étude.

$$
\text { Facteur } r(\mathrm{P}, t)
$$

Remarquons tout de suite à ce propos qu'une détermination physique de $r$ s'avérerait très difficile. Nous pouvons nous laisser guider ici par lintuition et par quelques considérations statistiques générales.

Nous verrons dans les applications numériques la forme définitive que nous avons adoptée. Il est bien entendu que cette forme pourrail se modifier pour d'autres bassins.

\section{Application de la méthode}

Nous avons profité de l'énorme effort d'équipement entrepris dans le bassin de la Haute-Durance pour mettre à l'épreuve la méthode développée plus haut.

Nous disposions en effet des limnigrammes originaux de la station de l'Archidiacre (B.V. $3.884 \mathrm{~km}^{2}$ ) depuis le mois de mai 1951 , ce qui nous permettait de suivre très complètement lallure des débits à cette station.

D'autre part, de nombreux postes pluviométri- ques enregistreurs furent installés durant l'année 1951 autour de la Durance si bien qu'il nous était possible également de suivre d'une façon continue l'évolution des averses sur les diverses parties du bassin.

L'application numérique a donné lieu à des recherches complémentaires concernant plus particulièrement la forme des fonctions $r$ et $k$, et le choix des unités.

Comme nous l'avons signalé plus haut, la détermination théorique de $r$ s'avérait difficile. Nous avons adopté la forme suivante qui semble en bon accord avec la réalité :

$$
r=t e^{-(t / 2)}
$$

On constate ainsi que nous faisons dépendie: uniquement de $t$ et non pas de $P$, ni des caractéristiques météorologiques. L'expérience nous a montré en effet, jusqu'à présent, qu'il s'établissait un régime moyen sur l'ensemble du bassin et qu'il était vain de chercher à différencier les différentes zones.

Le problème était plus simple pour $k$. En effet, nous savions déjà quelque chose a priori sur le coefficient : il est compris entre 0 et 1 , et il croît au cours du temps. Des considérations diverses sur la rapidité de l'absorption nous ont conduit à utiliser la forme suivante :

$$
k=1-e^{-a t}
$$

Nous avons obtenu ainsi une représentation très satisfaisante.

Pour lintégration numérique, il fallait en outre déterminer les valeurs de $\Delta \tau, \Delta t$ et $\Delta S$. Nous avons commencé par prendre $\Delta \tau=\Delta t$, ce qui nous a grandement simplifié les calculs. Le choix de cette valeur commune se trouvait lié à la grandeur du bassin, à la vitesse d'écoulement des eaux. Finalement, des considérations purement empiriques nous ont fait adopter six heures pour valeur commune de $\Delta \tau$ et de $\Delta t$.

Quant au $\Delta S$, nous ne lavons pas choisi constant, mais l'avons lié à la valeur commune de $\Delta t$ et $\Delta \tau$ par la méthode des temps de parcours égaux réalisée de la façon suivante : on trace sur le bassin une ligne fictive dont tous les points sont à une distance in $\Delta t$ de l'exutoire, distance mesurée le long des lignes de ruissellement de la pluie. Puis on trace la ligne dont tous les points sont à la distance $(i-1) v \Delta t$ de l'exutoire. Nous appellerons $h_{i j}$ la hauteur d'eau tombée sur la zone $\Delta S_{i}$ dans l'intervalle de temps $t_{0}+(j-1) \Delta t, t_{0}+j \Delta t$.

On établit alors facilement les feuilles de calculs qui donnent les apports de chaque zone. Pour la zone $\Delta S_{1}$, par exemple, nous aurons le tableau suivant : 


\begin{tabular}{|c|c|c|c|c|}
\hline $\begin{array}{l}\text { Volume } \\
\text { précipite }\end{array} \quad$ Périodes & $\Delta t_{1}$ & $\Delta t_{2}$ & $\Delta l_{3}$ & $\Delta t_{1}$ \\
\hline$h_{11} \cdot \Delta S_{1}=V_{11}$ & $r_{1} r_{11}$ & $r_{2} V_{11}$ & $r_{2: 3} V_{11}$ & $r_{1} V_{11}$ \\
\hline$h_{12} \Delta S_{1}=V_{12}$ & & $r_{1} V_{12}$ & $r_{2} V_{12}$ & $r_{3} V_{12}$ \\
\hline$h_{13} \cdot \Delta S_{1}=V_{13}$ & & & $r_{1} V_{13}$ & $r_{2}, V_{13}$ \\
\hline$h_{14} \cdot \Delta S_{1}=V_{14}$ & & & & $r_{1} V_{14}$ \\
\hline & & & & \\
\hline $\begin{array}{l}\text { Apports globaux } \\
\text { bruts de la zone I }\end{array}$ & $A_{11}^{\prime}$ & $A^{\prime}{ }_{12}$ & $A_{1::}^{\prime}$ & $A_{1+1}^{\prime}$ \\
\hline
\end{tabular}

Apports compte tenu de l'absorption :

$$
\begin{array}{llll}
\mathrm{A}^{\prime}{ }_{11} k_{1} & \mathrm{~A}_{12}^{\prime} k_{2} & \text { ネ. } & \ldots \ldots \\
\mathrm{A}_{11} & \mathrm{~A}_{12} & \text { \% } & \ldots \ldots
\end{array}
$$

Symboliquement nous avons représenté par $\Delta t_{i}$ la période $t_{0}+(i-1) \Delta t, t_{0}+i \Delta t . A_{1 i}$ est l'apport de la zone $\Delta S_{1}$ qui va ruisseler dans cette période.

De la même façon, la zone $\Delta S_{2}$ va fournir les apports suivants :

$$
A_{21} \quad A_{22} \quad A_{2 ; 3} \quad \ldots \ldots
$$

De même encore, la zone $\Delta S_{3}$ fournira les apports suivants :

$$
A_{31} \quad A_{32} \quad A_{33} \quad \cdots \cdots
$$

Finalement, les apports totaux à l'exutoire nous seront fournis par un dernier tableau où les $t_{i}$ ont la même signification que précédemment :

\begin{tabular}{|lr|c|c|c|c|}
\hline $\begin{array}{l}\text { Apports } \\
\text { des zones }\end{array}$ & Périodes & $\Delta t_{1}$ & $\Delta t_{2}$ & $\Delta t_{3}$ & $\Delta t_{4}$ \\
\hline Apport de la zone $\Delta \mathrm{S}_{1}$ & $\mathrm{~A}_{11}$ & $\mathrm{~A}_{1:}$ & $\mathrm{A}_{13}$ & $\mathrm{~A}_{14}$ \\
Apport de la zone $\Delta \mathrm{S}_{2 .}$ & & $\mathrm{A}_{21}$ & $\mathrm{~A}_{2.2}$ & $\mathrm{~A}_{23}$ \\
Apport de la zone $\Delta \mathrm{S}_{33}$ & & & $\mathrm{~A}_{31}$ & $\mathrm{~A}_{32}$ \\
etc... & & & & \\
Apports totalux....... & $\mathrm{V}_{1}$ & $\mathrm{~V}_{2}$ & $\mathrm{~V}_{3}$ & $\mathrm{~V}_{4}$ \\
\hline
\end{tabular}

Il nous suffit ensuite de diviser les différents volumes $\mathrm{V} i$ par $\Delta t$ pour avoir les débits moyens instantanés durant les différentes périodes.
On trouvera fig. 2, 3 el + les résultats d'ajustement sur les crues d'octobre 1952, octobre 1953 et août 1954 .

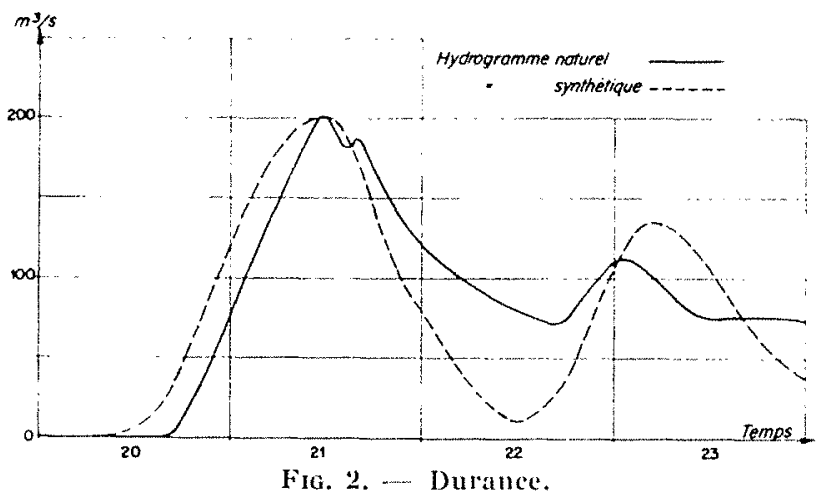

Station de jaugeage de l'Archidiace. Crue d'octobre 1952.

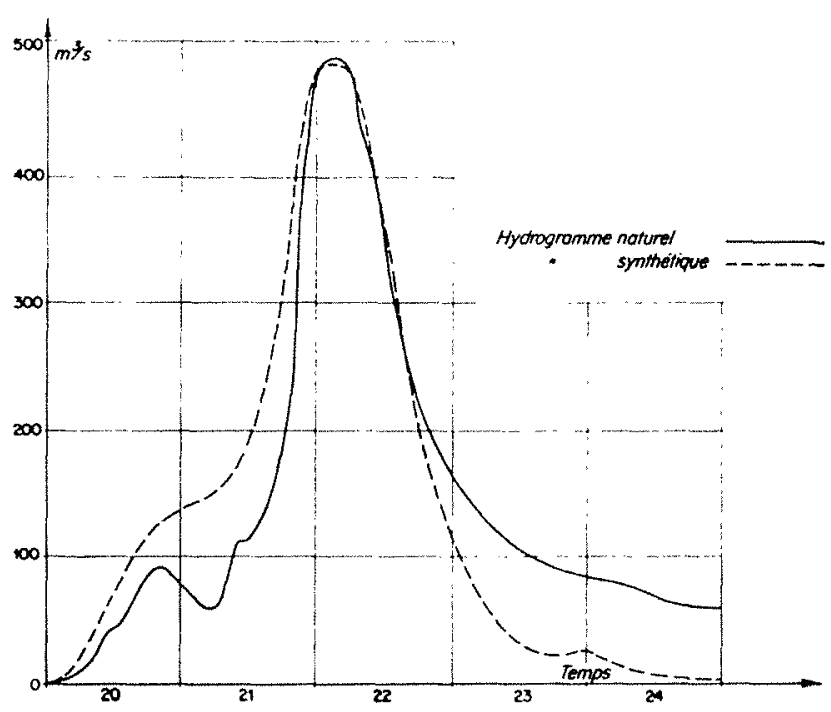

Fici. 3. - Durance.

Station de jaugeafe de l'Archidiacre. Cirue d'octobre 1953.

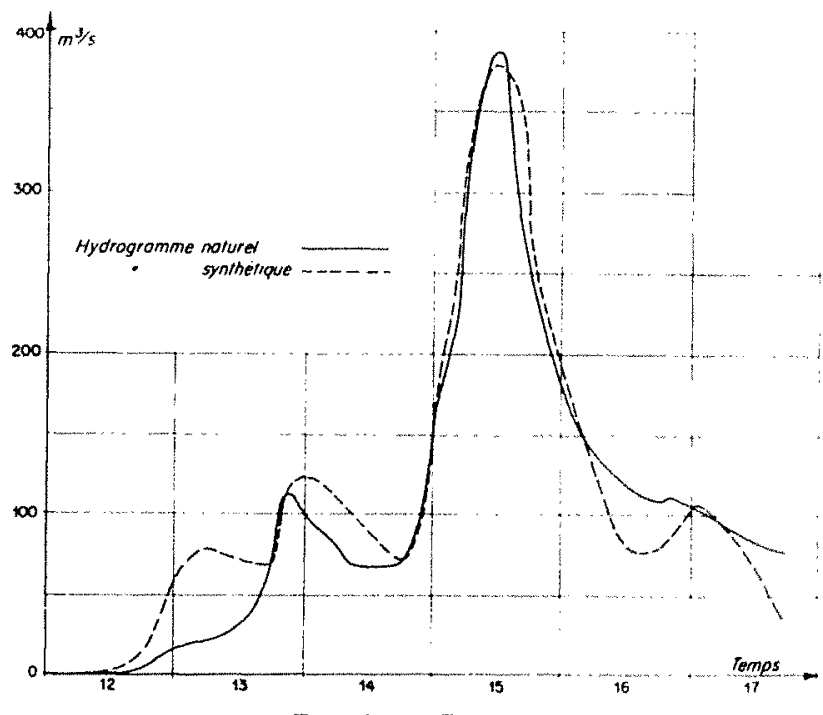

Fig. 4. - Durance.

Station de jaugeage de l'Archidiacre. Crue d'aout 1954. 
Les applications numériques que nous avons faites donnent lieu à un certain nombre d'observations.

On remarque tout d'abord que, systématiquement, l'hydrogramme synthétique se place audessus de l'hydrogramme naturel au début de la crue et en dessous à la fin. Cela ne doit pas nous surprendre outre mesure.

Au début de la crue, en effet, l'absorption est extrêmement importante et notre coefficient de ruissellement n'en tient pas suffisamment compte. Il serait possible, évidemment, d'introduire un terme correctif au début de la crue, mais sa détermination est trop artificielle pour offrir un intérêt suffisant dans l'état actuel de la question.

D'autre part, notre hydrogramme synthétique est systćmatiquement trop faible en fin de crue. Là encore, on peut trouver une explication simple à ce phénomène.

En effet, pour la détermination de l'hydrogramme naturel, pluie ruisselée, nous avons retranché de l'hydrogramme total la courbe de tarissement $\mathrm{AB}^{\prime}$ (voir fig. 1). Il s'ensuit évidemment que notre hydrogramme n'est pas le résultat du seul ruissellement, mais qu'il intègre également les apports de la nappe phréatique et du ruissellement hypodermique. Nous avons renoncé à tracer une courbe moyenne entre $A B^{\prime}$ et $A^{\prime} B$ en raison de la grosse part d'arbitraire qui entre dans cette détermination. Au contraire, par sa construction mème, l'hydrogramme synthétique n'utilise que les apports de ruissellement, d'où l'écart observé.

Naturellement, certains points de détail pourront être repris ultérieurement. C'est d'ailleurs là une tâche que nous poursuivrons.

\section{*}

Les remarques que nous venons de faire quant à l'écart entre hydrogramme naturel et hydrogramme synthétique nous ont suggéré une méthode de détermination pour la courbe délimitant le volume relatif au ruissellement pur dans l'hydrogramme naturel.

On pourrait en effet construire cette courbe $C$ en faisant coïncider la queue de l'hydrogramme réel $R$ avec celle de l'hydrogramme synthétique $S$ supposé a priori représenter correctement le ruissellement. La courbe $C$ ne serait pas autre chose que $R-S$.

Naturellement, il faudra mettre la méthode longtemps à l'épreuve avant de pouvoir accorder quelque confiance à ce procédé.

Nous pensons toutefois que la méthode peut ainsi servir non seulement à représenter les crues, mais encore à les analyser. Il y aura là, peut-être, un moyen de raccorder la théorie des crues pluviales à celle des courbes de tarissement. Nous attaquerons cette question dans un avenir que nous souhaitons proche.

\section{DIS CUSSION}

président : M. Hupwers

M. le Président remereie M. Lammev et lui demande si son étude est applicable aux crues de printemps dues en partic à la fonte de la neige.

M. Lanneu répond qu'il n'a pas eu l'occasion de faire une telle application, car les crues recentes de la Durance n'ont pas eu lien au printemps, mais il pense que, vu la nature des choses, il suffirait dajouter a sa formule un terme correctif.

M. le Président rapproche les formules de décroissance exponentielle utilisées par $M$. Larniev de celles que II. Magnin a employées dans son étude des crues de la Dordogne à Marèges.

M. Luarez considère l'étude comme particulièrement intéressante en tant qu'essai d'interprétation d'un phénomène naturel complexe. Il demande si, dans le cas concret de la Durance, les donnces propres au bassin, en particulier les temps de concentration d'une part et l'importance des calculs d'autre part, font que la méthode est applicable à la prévision pratique des crues.

M. Larniey estime qu'un simple calculateur pourra prédire la crue avant qu'elle se produise, à condition de connaitre les situations météorologiques, et de préparer a lavance des tableaux pour le calcul; ceci constituera la partie la plus longue du travail. On pourra d'ailleurs ctablir un catalogue des crues possibles en construisant les hydrogrammes synthétiques relatifs aux différents types d'averses qu'on peut envisager. Chaque hydrogramme synthétique sera répertorié en fonction des conditions météorologiques susceptibles de l'avoir produit, et la prévision de la crue consistera à choisir dans le catalogue ainsi constitué l'hydrogramme synthétique correspondant aux conditions météorologiques rencontrées effectivement.

M. Remennenas voudrait que l'on corrige la solution analytique par un coup de pouce tenant compte des caractéristiques physiques de la zone étudiée et de la saison durant laquelle survient la crue : il est visible, en effet, que les premières pluies d'octobre donnent, toutes choses egales d'ailleurs, moins de débil que celles de printemps.

M. Magnin s'associe à la remarque de M. Rementeras et suggère de rajuster le coeffici nl de ruisellement suivant le débit d'origine.

M. LaRmeu répond que le paramètre de décroissance a résultant du rajustement des crues étudiées était de 0,025 pour la première crue (celle d'octobre 1952); 0,028 pour la crue d'octobre 1953 et 0,030 pour la crue d'aoút 1954. Les coefficients de ruissellement respectifs sont de 15,17 et $20 \%$ : il serait loisible d'étudier des crues de printemps et de choisir le coefficient qui correspond à une époque considérée. 
M. Duranton remarque que pour le dimensionnement des évacuateurs de crue, cc sont essentiellement les très forts débits qui sont intéressants et par suite :

a) On utilise surtout la partie superieure des courbes hydrogrammes (pointes de crues);

b) Les crues catastrophiques à envisager supposent des circonstances exceptionnelles et notamment un coefficient de ruissellement élevé (sol saturé).

Par consequent, on doit arriver à des résultats très convenables pour la partie supérieure de l'hydrogramme, qui nous intéresse.

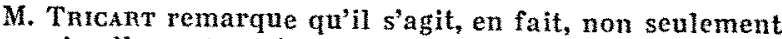
du ruissellement stricto sensu mais de l'ensemble de l'ćcoulement, comprenant le ruissellement superficiel, l'infiltration, la recharge des nappes, et le debit des sources: ces trois phases de l'ćcoulement sont décalées entre elles dans le temps et la forme de chacune delles dépend de la nature du sol, de sa pente, de la couverture véqútale, etc... Bien que ces éléments soient intégrés stafistiquement sous la forme de la fonction de répartition dans la formule proposée par M. Lannieu, une analyse de l'influence respective de chacun de ces facteurs, si elle est possible et point trop conteuse, permelleait de les introduire dans les formules et réduirait certainement les ecarts entre l'hydrogramme et la courbe experimentale, en meme temps qu'elle apporterait une conlribulion a la question des débits solides.

M. Chapouther voudrait etre certain qu'on est mieux documenté sur la pluie que sur les débits, sans quoi il paraitrait plus simple d'installer une station de jau geage sur une rivière que de multiplier les pluviomètres.

M. Lanrieu répond que l'hydrogramme est cependant indispensable pour la détermination des débits de cerlaines crues pour lesquelles les stations de jaugeage ne sont pas tarées, soit que les hauteurs depassent les normes de la station, soit que celle-ci ait éce emportée par le fot.

M. Bonsin indique qu'on peut determiner rapidement le coefficient de répartition $\mathrm{R} / \mathrm{\vartheta}$ en mesumant le temps du trajet des molécules d'eau au moyen d'injections de nuorescéine.

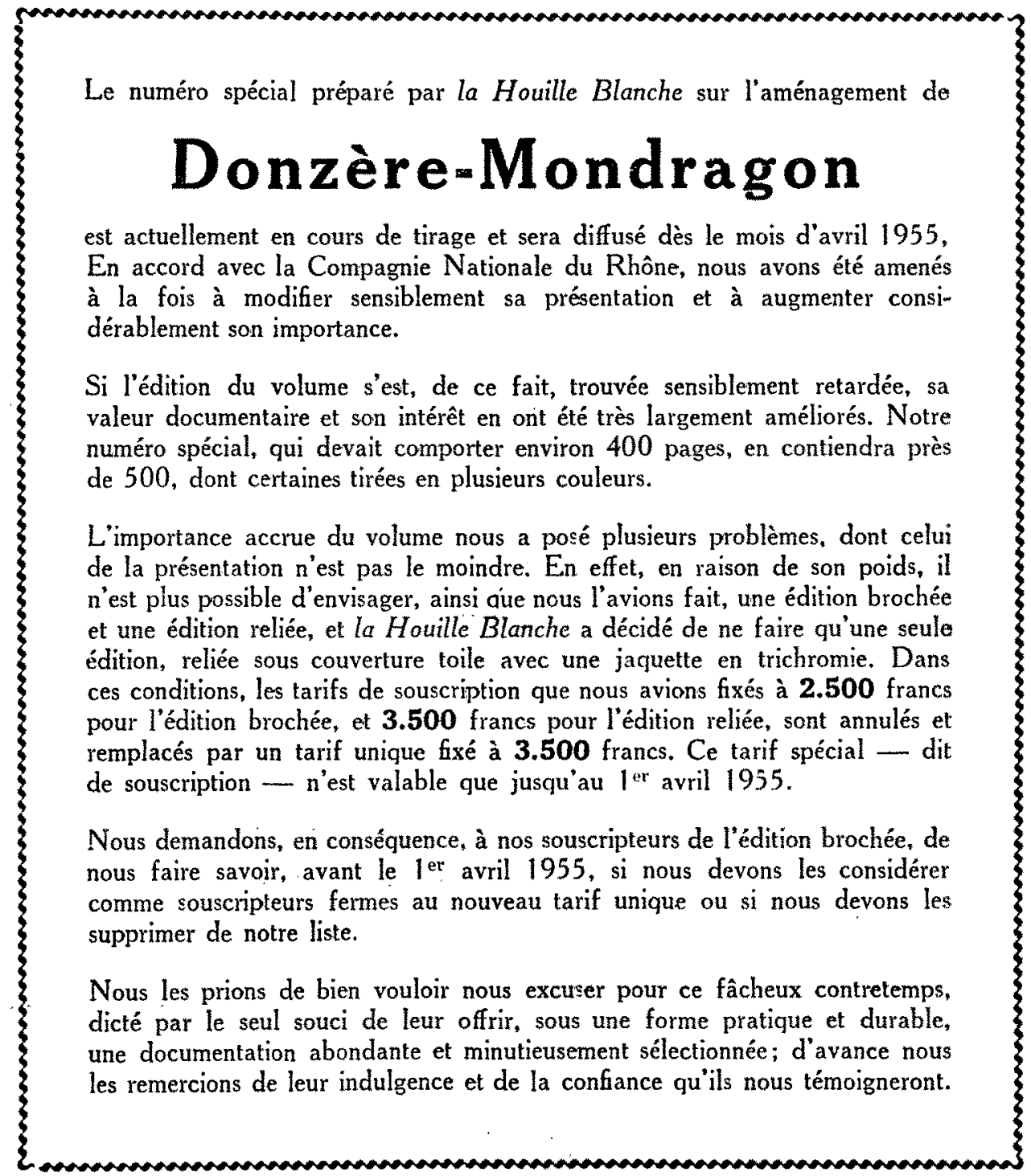

